\title{
Incidence, clinical manifestations and clipping of nail psoriasis in the dermatology center of the Hospital Universitário Evangélico de Curitiba*
}

\author{
Luiz Eduardo Fabricio de Melo Garbers ${ }^{1}$ \\ Lincoln Helder Zambaldi Fabricio ${ }^{1}$ \\ Aguinaldo Bonalumi Filho ${ }^{3}$
}

\author{
Helena Slongo ${ }^{1}$ \\ Juliano Vilaverde Schmitt ${ }^{2}$
}

DOI: http:/ / dx.doi.org/10.1590/abd1806-4841.20164296

\begin{abstract}
BACKGROUND: Psoriasis is a chronic inflammatory skin disease that often progresses with nail alterations. It is suspected that there is a correlation between nail psoriasis and enthesitis of the distal interphalangeal joint, seeming to serve as a predictor.

Oвјестіves: To analyze the profile of patients with nail psoriasis and correlate the presence of nail alterations with psoriatic arthritis, quality of life, extent of psoriasis and the histopathology of the nail.

MetHods: An observational cross-sectional study with 40 patients with a diagnosis of psoriasis and without systemic treatment. The patient profile was researched, including quality of life and evaluated for the presence of psoriatic arthritis. The severity of the skin psoriasis and the presence of nail lesions were evaluated. Nail fragments were collected and analyzed through clipping. It obtained $100 \%$ of positivity for psoriasis in the histopathology exam of the nail plate.

REsULTS: Of the 40 patients, $65 \%$ were diagnosed with nail psoriasis. Suggestive findings of psoriatic arthritis in hands were present in $33 \%$, being more frequent in those with nail alteration $(p=0.01)$. In $92.3 \%$ of patients diagnosed with psoriatic arthritis in the hands there was some nail injury. The most frequent injuries were pitting and onycholysis.

CONCLUSIONS: Patients with nail psoriasis are usually men, with worse quality of life and higher chance of psoriatic arthritis. The correlation between the nail involvement of psoriasis and psoriatic arthritis in hands confirms the association between these two forms. The clinical diagnosis of nail psoriasis did not correlate with the histological diagnosis.
\end{abstract}

Keywords: Arthritis, psoriatic; Indicators of quality of life; Nail diseases; Psoriasis; Quality of life

\section{INTRODUCTION}

Psoriasis is a chronic inflammatory disease, noncontagious, which leads to the appearance of erythematous scaly lesions on skin. ${ }^{1}$ Its annual incidence doubled between 1970 and 2000 and its worldwide prevalence is estimated to be approximately $2-3 \%{ }^{2}$

The etiology of psoriasis is not fully understood but it is known that its pathogenesis involves genetic, immunological and environmental interaction. ${ }^{3}$ It is associated with the presence of HLA-B57 and HLACw6 antigens and one third of patients have a positive family history. ${ }^{4}$

The most troublesome symptoms are rash, joint pain due to psoriatic arthritis, constant peeling skin and pain. ${ }^{5}$ The body regions most frequently affected are the scalp, knee and elbow. ${ }^{6}$ 
The diagnosis of cutaneous psoriasis is essentially clinical, by history and physical examination, revealing the Auspitz's sign. The diagnosis can be confirmed anatomopathologically. ${ }^{7}$

Approximately $20 \%$ of patients with psoriasis also have psoriatic arthritis, a rheumatic-inflammatory joint disease that develops years after the onset of psoriasis, although it may occur at any time: in rare cases it occurs before psoriasis or even isolated. ${ }^{8}$

Psoriatic arthritis is essentially oligoarticular, asymmetric, reaching the small joints of the hands, typically the distal interphalangeal joint. Inflammation also affects ligaments and muscles, and it may lead to enthesitis. ${ }^{9-11}$

Diagnosis of psoriatic arthritis is established by the CASPAR criteria (Classification Criteria for Psoriatic Arthritis), which assess the presence of psoriasis, nail dystrophy, negative rheumatoid factor, dactylitis and radiological findings in a justarticular bone neoformation. ${ }^{12}$

Nail psoriasis is a very common form of psoriasis activity, $50 \%$ of diagnosed patients present the disease on the nails. ${ }^{13}$ It is rare to find it alone, only $1 \%$ to $5 \%$ of nail psoriasis are not accompanied by skin manifestations. ${ }^{14}$

Clinically, psoriasis in the nail plate presents onycholysis, oil stains, subungual hyperkeratosis, and splinter hemorrhage; and, in the nail matrix, pitting, leukonychia, crumbling and red spots on the lunula. ${ }^{15}$

The Nail Psoriasis Severity Index (NAPSI) was developed as an attempt to find an algorithm for classification and treatment of this entity. This classification divides each nail into 4 quadrants and evaluates the presence of manifestations of psoriasis in the matrix and plate. ${ }^{11}$

Nail injury is strongly associated with psoriatic arthritis, especially with the distal interphalangeal joint. Approximately $80 \%$ of patients with psoriatic arthritis present nail psoriasis. ${ }^{16}$ Studies using high resolution ultrasound and nuclear magnetic resonance showed that the nail is part of the muscle-skeletal appendix, being connected to the distal interphalangeal joint both anatomically and functionally, and that patients with onychopathies have more abnormalities of the distal interphalangeal joint seen in imaging. ${ }^{17,18}$ Many authors consider the enthesitis as the center of the inflammatory process leading to effects on cartilage and bone, as well as in the matrix and nail bed. Studies also show association between severity of nail disease and joint disease. ${ }^{19}$

The nail involvement, due to associated inflammation of the distal interphalangeal joint, can serve as a predictor of skin psoriasis severity or as a psoriatic arthritis precursor, besides causing pain, psychological stress and reduced manual dexterity, damaging professional, functional and social conditions. ${ }^{20}$

\section{METHODS}

Observational cross-sectional study conducted at a dermatology clinic of the Hospital Universitário de Curitiba, approved by the Ethics Research Committee. The study included patients followed at the dermatology clinic with the diagnosis of psoriasis already established, without systemic treatment for six months, suitable and in accordance with the terms of consent.

Patients were assessed thought questionnaires, which analyzed the type of psoriasis, age of first manifestation of the disease, sex, alcoholism and smoking. Arthritis with history and physical examination showing worsening of pain at rest, improving with physical exercise and throughout the day, and that was more intense in the early hours of the day, was considered joint involvement of the inflammatory type, representative of psoriatic arthritis, excluding cases associated with other rheumatic diseases.

The impact of psoriasis on quality of life of the patient was examined by Dermatology Life Quality Index (DLQI), considered severe when above 10. Psoriasis severity is assessed using Psoriasis Area and Severity Index (PASI) according to the method described originally by Fredriksson and Petters.

To evaluate the presence of nail disease we used an adapted Nail Psoriasis Severity Index (NAPSI), evaluating macroscopically the nail plate to search for the presence of onycholysis, oil stain, subungual hyperkeratosis and splinter hemorrhage; and the nail matrix to search for pitting, leukonychia, crumbling and red spots on the lunula.

Clipping, a method in which a fragment of the distal part of the nail plate is cut for histopathological evaluation, was performed in the most affected chirodactyl nail of patients who presented macroscopic nail psoriasis. In patients without nail changes in the physical examination, the clipping was performed in the fourth chirodactyl of the right hand.

The fragment cut from the distal part of the affected nail should be at least $5 \mathrm{~mm}$ horizontally and placed in an empty vial. All nail properly acquired after the histological method were evaluated on hematoxylin-eosin by the same pathologist of the hospital.

The parakeratosis observed histologically was classified as extensive or focal. Extensive was divided into severe, moderate and mild. Nail psoriasis was determined in reports that found nail plate with extensive parakeratosis occupying its entire length and thickness and absence of fungi.

Continuous variables were represented by mean and standard deviation (SD), or medians and interquartile deviations (IQD) and analyzed bi variously by parametric Student's t-test student or non-parametric Mann-Whitney test, depending on the nor- 
mality of distributions, assessed by Shapiro-Wilk test. Categorical variables were compared by chi-square or Fisher's exact test, according to the expected frequency less than or greater than 5 to one or more cells in each analysis. Correlations were analyzed by Pearson or Spearman tests, according to the normality of distributions. The categorical associations were represented by the prevalence ratios and $95 \%$ confidence intervals (95\% CI).

The influence of nail involvement extension, PASI, age and sex on the DLQI (dependent variable) was analyzed in a multivariate way using multivariate linear regression with inclusion of variables by the "stepwise" method with input criteria of $p<0.3 .{ }^{21}$

We considered significant two-tailed $p$ values $<0.05$. Data were tabulated by Windows Office Excel 2013 and analyzed by IBM SPSS 21.

\section{RESULTS}

We assessed 40 patients, respecting the inclusion and exclusion criteria mentioned above. Of these patients, 25 (62.5\%) were women and $15(37.5 \%)$, men.

Mean age of patients was 50.7 years $(\mathrm{SD}=12.2)$, and disease onset occurred at mean age of 37.4 years $(\mathrm{SD}=12.0)$. The most common clinical forms were vulgar $(n=29 ; 73 \%)$ and palmoplantar $(n=5 ; 13 \%)$, with 1 case of guttate psoriasis, 1 of pustular psoriasis, 2 cases of inverse psoriasis and 2 of erythrodermic psoriasis.

Nail disease was diagnosed in 26 patients $(65 \%)$, following the impairment criteria of at least one chirodactyl, according to NAPSI.

Number of nails altered per patient was significantly higher in men $(10$ [IQD $=6$ ] versus 4 [IQD = 2]; $p=0.029$ - Mann-Whitney), with nail involvement presenting a median disease evolution time of 5 years (IQD = 10 years).

Joint involvement of the hands of inflammatory type, suggestive of psoriatic arthritis by history and physical examination, was presented in 13 patients $(32.5 \%)$, being more frequent in those with nail changes $(46.2 \%$ vs $7.1 \%$; PR $=1.78 ; 95 \%$ CI: 1.20 to $2.64 ; \mathrm{p}=$ 0.015 - Fisher's exact test).
Compared with patients with nail changes by the NAPSI criteria, the matrix was affected in 19 $(73.1 \%)$ and the bed in $23(88.5 \%)$ patients. There was an association between the occurrence of joint pain and matrix involvement ( $\mathrm{PR}=2.31 ; 95 \% \mathrm{CI}: 1.25$ to 4.25; $\mathrm{p}=0.017$ - Fisher's exact test) but not with bed involvement ( $\mathrm{PR}=1.60 ; 95 \% \mathrm{CI}: 0.98$ to $2.61 ; \mathrm{p}=0.103$ - Fisher's exact test).

The most frequent nail changes were pitting, onycholysis and hyperkeratosis (Table 1). The findings of psoriatic nail involvement didn't favor any chirodactyl in particular ( $p=0.568$ - chi-square grip), with the following proportion of involvement from the first to the fifth fingers: $53.8 \%, 48.1 \%, 55.8 \%, 65.4 \%$ and $42.3 \%$

Mean DLQI was $10.45(\mathrm{SD}=6.86)$ and the median PASI was $3(\mathrm{IQD}=4)$ with a significant correlation between these variables (Spearman's Rho $=0.41 ; \mathrm{p}=$ 0.010) (Table 2).

The number of affected nails didn't correlate linearly with PASI (Spearman's Rho $=0.23 ; \mathrm{p}=0.170$ ), but patients with PASI greater than or equal to 10 presented a higher number of affected nails (5 [IQD $=0$ ] vs. 2 [IQD = 3]; $\mathrm{p}=0.006$ - Mann-Whitney). The extent of nail involvement correlated with DLQI (Spearman's Rho $=0.43 ; p=0.007)$. Patients with 5 or more changed nails presented mean DLQI of $16.2(\mathrm{SD}=7.7)$ versus $7.7(S D=4.5)$ of those with less affected nails $(p$ $=0.002-$ Student's t-test).

TABLE 1: Nail changes presented by NAPSI

\begin{tabular}{lll}
\hline Nail changes by NAPSI & Cases & $\%$ \\
\hline Pitting & 17 & 65 \\
Onycholysis & 17 & 65 \\
Hyperkeratosis & 14 & 54 \\
Crumbling & 10 & 38 \\
Oil slick & 9 & 35 \\
Leukonychia & 5 & 19 \\
Splinter & 3 & 12 \\
Red spot & 1 & 4 \\
\hline
\end{tabular}

TABLE 2: Linear regression evaluating the effect of variables on the DLQI.*

\begin{tabular}{lclll}
\hline $\begin{array}{l}\text { Variable } \\
\text { Coefficient }\end{array}$ & 95\% Confidence interval & Beta & P value & \\
\hline Age & -0.13 & -0.27 to 0.01 & -0.23 & 0.069 \\
PASI & 0.24 & 0.07 to 0.40 & 0.39 & 0.006 \\
Number of affected nails & 0.63 & 0.11 to 1.16 & 0.33 & 0.019 \\
Constant & 13.44 & 5.76 to 21.13 & - & 0.001 \\
\hline
\end{tabular}

${ }^{*} \mathrm{R} 2=0.44 ; \mathrm{F}=9.480(\mathrm{GL}=3) ; \mathrm{p}($ model $)<0.001 ; \mathrm{n}=40$. DLQI: Dermatology Life Quality Index. PASI: Psoriasis Area Severity Index. Dependent variable: DLQI. 
In the multivariate analysis we found that there is a significant linear association between DLQI and explanatory variables age, PASI and number of affected nails ( $p<0.001)$. Furthermore, from the coefficient of determination $\left(\mathrm{R}^{2}=0.44\right)$ we can say that $44 \%$ of DLQI variations are explained by these variables. The estimated coefficient for PASI variable was equal to 0.24 with statistical significance $(p=0.006)$. Thus, for each extra unit of PASI, we estimate an increase of 0.24 units in DLQI, keeping fixed the other variables included in the model (age and number of affected nails). There is $95 \%$ of confidence that the interval $(0.07 ; 0.40)$ contains the referred increase for each extra unit of PASI. Regarding the number of affected nails, the estimated coefficient was $0.63(p=0.019)$. This result indicates that for each most affected nail it is estimated an increase of 0.63 units in DLQI, keeping fixed the other variables included in the model (age and PASI). There is 95\% of confidence that the interval $(0.11 ; 1.16)$ contains the referred increase for each extra affected nail.

In clipping, $100 \%$ of patients presented histological finding of parakeratosis, 35 (87.5\%) had extensive parakeratosis and $5(12.5 \%)$ had focal parakeratosis. Other alterations found were subungual keratosis $(22.5 \%)$, slots $(20 \%)$ and serous lakes $(12.5 \%)$. No fungus was found (Tables 3 and 4) (Figure 1).
TABLE 3: Histopathological findings by CSME

\begin{tabular}{lll}
\hline Histopathological findings & Cases & \% \\
\hline Extensive parakeratosis & 35 & 87.5 \\
Subungueal keratosis & 9 & 22.5 \\
Slots & 8 & 20.0 \\
Focal parakeratosis & 5 & 12.5 \\
Serous lakes & 3 & 7.5 \\
\hline
\end{tabular}

Reports of clippings by the pathology department of the hospital. Presence fungi was found in no nail bed

TABLE 4: Histopathological findings in relation to parakeratosis

\begin{tabular}{lll}
\hline Results in relation to parakeratosis & Cases & \% \\
\hline Marked extensive parakeratosis & 31 & 77.5 \\
Moderate extensive parakeratosis & 3 & 7.5 \\
Discreet extensive parakeratosis & 1 & 2.5 \\
Focal parakeratosis & 5 & 12.5 \\
\hline
\end{tabular}

$100 \%$ of reports showed presence of parakeratosis.
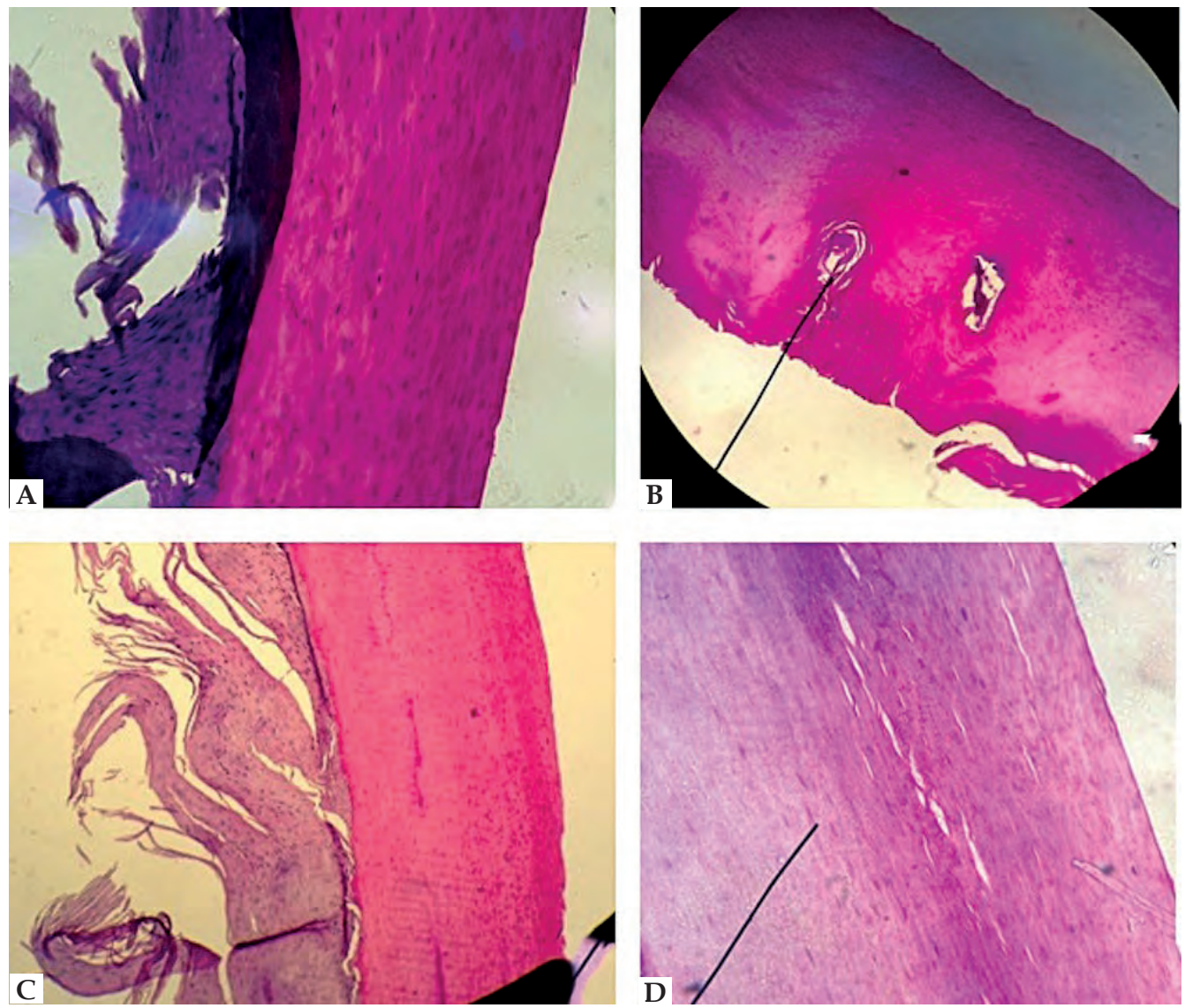

Figure 1:

A. Marked extensive parakeratosis.

B. Serous lake.

C.S u bu n g u a 1

keratosis.

D. Slots 
Histological diagnosis of nail psoriasis, defined as extensive parakeratosis and absence of fungi, was presented in 35 patients, but there was no significant association with the clinical diagnosis of nail psoriasis ( $p=0.322$ - Fisher's exact test). By contrast, $78.6 \%$ of patients without nail lesions on physical examination showed histological diagnosis of nail psoriasis.

Only the presence of marked extensive parakeratosis tended to be associated to clinical diagnosis (PR $=2.58 ; 95 \%$ CI: 1.21 to $5.50 ; \mathrm{p}=0.044-$ Fisher's exact test), especially in the presence of matrix involvement $(\mathrm{PR}=2.12 ; 95 \% \mathrm{CI}: 1.32$ to $3.41 ; \mathrm{p}=0.021-$ Fisher's exact test).

Considering the clinical examination as a reference in the diagnosis of nail psoriasis, the histological finding of marked extensive parakeratosis had a sensitivity of $88.5 \%$, but a specificity of $42.9 \%$ compared with other psoriatic patients without clinically evident nail involvement.

The histological diagnosis of nail psoriasis was not associated with the presence of arthritis in the hands (PR $=0.89 ; 95 \%$ CI: 0.64 to 1.24; $p=0.584$ - Fisher's exact test).

\section{DISCUSSION}

In this study we found nail change in $65 \%$ of 40 studied cases of psoriasis. This proportion is higher than that reported by a German study $(40.9 \%)$ and almost identical to that reported by a French study $(61 \%))^{22,23}$

Considering all patients with nail psoriasis in the study, the number of changed nails was higher in men. This difference greater than $10 \%$ between men and women has also been reported by other studies, noting that psoriasis generally has no gender preference. ${ }^{11,22}$

Among the clinical presentations of the most frequent nail lesions were pitting and onycholysis, present in $65 \%$ of cases. This result met our expectations. ${ }^{20}$

We found a strong statistical correlation between nail involvement and joint pain of inflammatory type suggesting that patients with enthesitis of the distal interphalangeal joint develop psoriatic arthritis and nail lesions more frequently, especially when the changes are related to the nail matrix. Of the 13 pa- tients with psoriatic arthritis, 92.3\% had nail psoriasis.

DLQI score was higher, which represents a poorer quality of life, in patients with nail lesions, and there is a positive correlation between the number of affected nail and impairment of quality of life, with an increase of 0.63 points in DLQI for each affected nail. This finding was independent of PASI and was also observed in other studies. ${ }^{24}$ Moreover, we observed significantly more extensive nail involvement in cases with higher PASI, which was previously reported. ${ }^{22,23,25,26}$

Regarding histopathological findings, $100 \%$ of patients presented parakeratosis and absence of fungi in the sample. Diagnosis of nail psoriasis by histology was not related statistically to diagnosis by macroscopic examination of the nail, only about the description of marked extensive parakeratosis.

This result can be explained by two factors: first, the clipping can identify with precocity the psoriasis manifestations that are not found clinically. Added to this fact, we know that the pathological changes of nail growth occur from the matrix toward the distal nail bed within approximately three months, thus only if the patient's condition remain unaffected during this period we will have the perfect agreement between the CSME and presented clinic.

\section{CONCLUSION}

Nail involvement is an important manifestation of psoriasis and it is associated with a much more severe disease both in the articular and in the impact on quality of life point of view. The nail disease seems to influence the patient's quality of life regardless of the extent of skin involvement. And apparently it is more extensive among men.

The association between involvement of the nail matrix in the psoriasis and psoriatic arthritis in the hands strengthens the hypothesis of a relation between these two manifestations of the disease.

The most common nail manifestations of psoriasis are pitting in onycholysis.

Histological examination of psoriatic patients' nails often presents parakeratosis, even in the absence of clinically significant alterations.] 


\section{REFERENCES}

1. Langham S, Langham J, Goertz HP, Ratcliffe M. Large-scale, prospective, observational studies in patients with psoriasis and psoriatic arthritis: a systematic and critical review. BMC Med Res Methodol. 2011;11:32.

2. Schon P, Boehncke WH. Psoriasis. N Engl J Med. 2005;352:1889-1912.

3. Mehta NN, Azfar RS, Shin DB, Neimann AL, Troxel AB, Gelfand JM. Patients with severe psoriasis are at increased risk of cardiovascular mortality: A cohort study using the General Practice Research Database. Eur Heart J. 2010;31:1000-6.

4. Sanchez APG. Imunopatogênese da psoríase. An Bras Dermatol. 2010;85:747-9.

5. Globe D, Bayliss MS, Harrison DJ. The impact of itch symptoms in psoriasis: results from physician interviews and patient focus groups. Health Qual Life Outcomes. 2009;7:62.

6. Torpy JM, Burke AE, Golub RM. Psoriasis. JAMA. 2011;306:896.

7. National Psoriasis Foundation. An overview of psoriasis and psoriatic arthritis. Portlan: Psoriasis Foundation; 2009

8. Myers WA, Gottlieb AB, Mease P. Psoriasis and psoriatic arthritis: clinical features and disease mechanisms. Clin Dermatol. 2006;24:438-47.

9. Myers A, Kay LJ, Lynch SA, Walker DJ. Recurrence risk for psoriasis and psoriatic arthritis within sibships. Rheumatology (0xford). 2005;44:773-6.

10. Mrowietz U, Reich K. Psoriasis - new insights into pathogenesis and treatment. Dtsch Arztebl Int. 2009;106:11-8.

11. Reich K. Approach to managing patients with nail psoriasis. J Eur Acad Dermatol Venereol. 2009;23:15-21.

12. Taylor W, Gladman D, Helliwell P, Marchesoni A, Mease P, Mielants H, et al. Classification criteria for psoriatic arthritis: development of new criteria from a large international study. Arthritis Rheum. 2006;54:2665-73.

13. Jiaravuthisan MM, Sasseville D, Vender RB, Murphy F, Muhn CY. Psoriasis of the nail: anatomy, pathology, clinical presentation, and a review of the literature on therapy. J Am Acad Dermatol. 2007;57:1-27.

14. Van Laborde S, Scher RK. Developments in the treatment of nail psoriasis, melonychia striata, and onychomycosis. A review of the literature. Dermatol Clin. 2000;18:37-46.

15. Farias DC, Tosti A, Chiacchio ND, Hirata SH. Dermoscopy in nail psoriasis. An Bras Dermatol. 2010;85:101-3.

16. Lawry M. Biological therapy and nail psoriasis. Dermatol Ther. 2007;20:60-7.

17. Scarpa R, Soscia E, Peluso R, Atteno M, Manguso F, Del Puente A, et al.Nail and distal interphalangeal joint in psoriatic arthritis. J Rheumatol. 2006;33:1315-9.

18. Machado APB, Ataíde D, Sandri C, Vandressen N, Jordão J. The importance of radiologic evaluation and physical examination in diagnosis of psoriatic arthritis and its prevalence in the Hospital Universitário Evangélico de Curitiba. An Bras Dermatol. 2005;80:S345-51.

19. Williamson L, Dalbeth N, Dockerty JL, Gee BC, Weatherall R, Wordsworth BP. Extended report: nail disease in psoriatic arthritis: clinically important, potentially treatable and often overlooked. Rheumatology (Oxford). 2004;43:790-4.

20. Baran R. The burden of nail psoriasis: an introduction. Dermatology. 2010;221:1-5.

21. Zachariae H, Zachariae R, Blomqvist K, Davidsson S, Molin L, Mørk C, et al. Quality of life and prevalence of arthritis reported by 5795 members of the Nordic Psoriasis Associations. Acta Derm Venereol. 2002;82:108-13.

22. Augustin M, Reich K, Blome C, Schäfer I, Laass A, Radtke MA. Nail psoriasis in Germay: epidemiology and burden of disease. Br J Dermatol. 2010;163:580-5.

23. Taieb C, Myon E, Voisard JJ, Marin N, Corvest M. Nail psoriasis: epidemiological study in France. EADV 2005. Poster P16.37.

24. Armesto S, Esteve A, Coto-Segura P, Drake M, Galache C, Martínez-Borra J, et al. Nail psoriasis in individuals with psoriasis vulgaris: a study of 661 patients. Actas Dermosifiliogr. 2011;102:365-72.

25. Maejima H, Taniguchi T, Watarai A, Katsuoka K. Evaluation of nail disease in psoriatic arthritis by using a modified nail psoriasis severity score index. Int $\mathrm{J}$ Dermatol. 2010;49:901-6.

26. Faria JR, Aarão AR, Jimenez LM, Silva $\mathrm{OH}$, Avelleira JC. Inter-rater concordance study of the PASI (Psoriasis Area and Severity Index). An Bras Dermatol. 2010;85:625-9.

\author{
MAILING ADDRESS: \\ Luiz Eduardo Fabricio de Melo Garbers \\ Alameda Augusto Stellfeld, 1908 \\ Bigorrilho \\ 80730-150 Curitiba - PR \\ Brazil \\ Email:legarbers@gmail.com
}

